\title{
ERRATUM
}

\section{Erratum to: Evaluation and Treatment of Gout as a Chronic Disease}

Fernando Perez-Ruiz · Ana Maria Herrero-Beites

(C) Springer Healthcare 2012

Erratum to: Perez-Ruiz F, Herrero-Beites AM. Evaluation and Treatment of Gout as a Chronic Disease. Adv Ther. 2012;29(11):935-46.

The authors of the above-mentioned paper would like to make the following adjustments to their article.

In the published Fig. 1, the values in the allopurinol $300 \mathrm{mg}$ once daily and febuxostat $120 \mathrm{mg}$ once daily columns are incorrect. The correct values are detailed in the corrected Fig. 1, below.

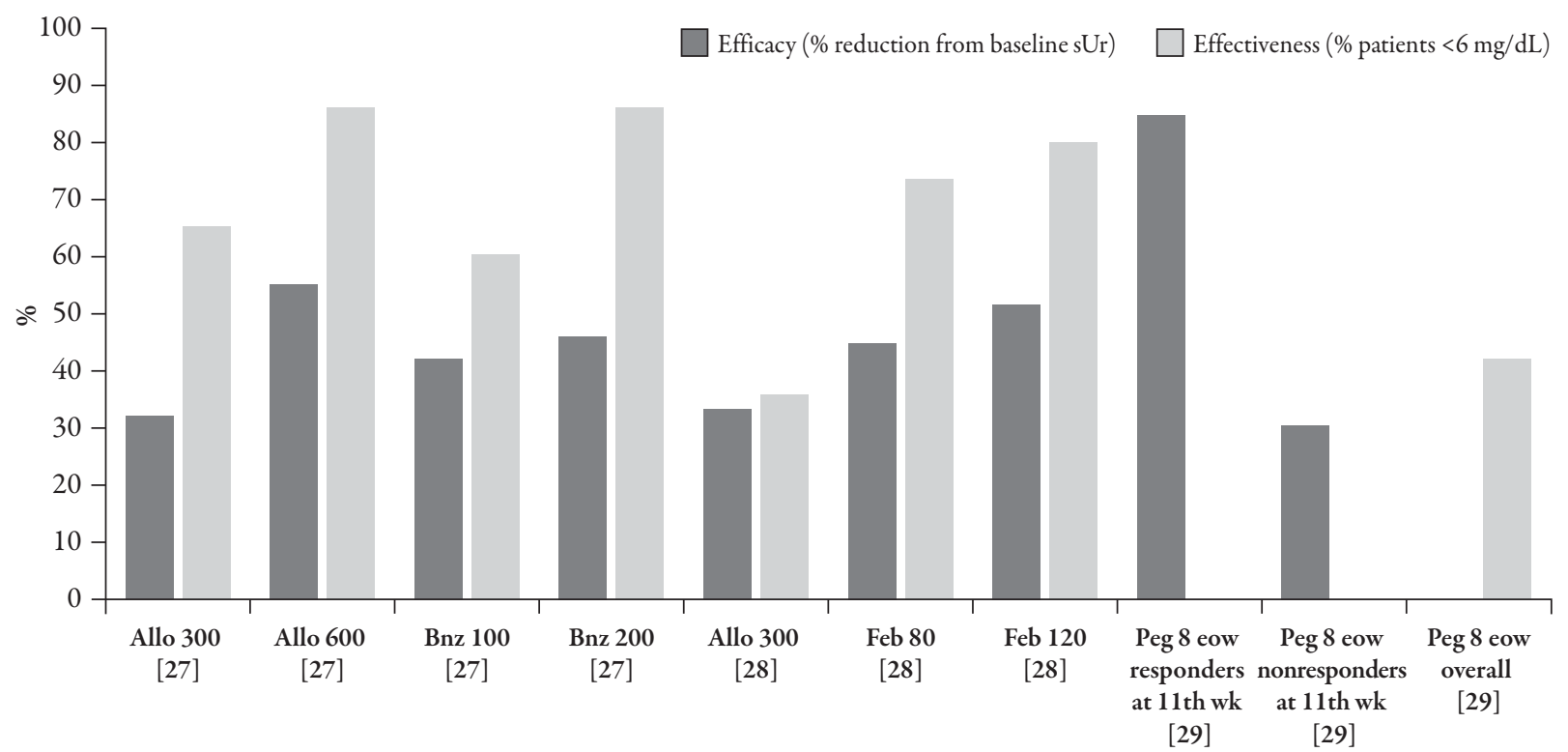

Fig. 1 Efficacy and effectiveness of ULDs in phase 3/4, double-blind, randomized trials. Head to head comparisons only for same references, otherwise indirect comparisons. Data of outcomes from final visit, except pegloticase: week 11 for efficacy and overall combined 3-6 months for effectiveness. Data obtained from [27-29]. Allo 300/600 allopurinol 300/600 mg once daily, Bnz 100/200 benzbromarone 100/200 mg/day, Feb 80/120 febuxostat 80/120 mg once daily, Peg 8 eow pegloticase $8 \mathrm{mg}$ biweekly, ULD urate-lowering treatment

The authors thank Springer Healthcare for publishing the correction. 